\title{
Intraocular Tuberculosis
}

\author{
P. H. ROSEN*, D. J. SPALTON and E. M. GRAHAM \\ London
}

\begin{abstract}
Summary
Twelve patients with a diagnosis of intraocular tuberculosis are described. Nine patients presented with florid ischaemic retinal vasculitis and a marked tendency to neovascularisation. Two patients developed choroidal tubercles; iris nodules were observed in association with anterior uveitis in the remaining patient. The methods of diagnosis, management and possible mechanisms of pathogenesis of these different clinical presentations are discussed.
\end{abstract}

Tuberculosis can affect any organ of the body. The initial infection usually occurs in the lung or alimentary tract and is characterised by a primary complex, which is the combination of a focal lesion with a caseating granuloma, and regional lymphadenopathy. Although primary lesions may rarely present in the conjunctiva or lids ${ }^{1}$ ocular tuberculosis is most likely to occur as part of a post primary infection due to direct haematogenous spread or by hypersensitivity responses. The different types of ocular involvement in tuberculosis are summarised in Table I: only intraocular tuberculosis is discussed here.

The incidence of tuberculosis in the United Kingdom has decreased markedly from 80,000 notifications in 1914 to 7,500 in $1982 .{ }^{2}$ By 1979 the annual rate per 100,000 in the UK was 10.7 for whites, 31 for West Indians and 382 for Asians, which emphasises the racial difference in incidence; tuberculosis remains very common in the third world.

Similarly, the implication of tuberculosis as a cause of uveitis has declined with the diminishing incidence of tuberculous infection and a better appreciation of other causes of uveitis. In Woods' series of $1944^{3} 52 \%$ of cases of uveitis were presumed to result from tubercu- losis, but Schlaegel in $1970^{4}$ found that this figure has reduced to $0.28 \%$ of cases.

The decline in incidence of the systemic disease has also resulted in a reduced emphasis on such a diagnosis by ophthalmologists. The overall incidence of ocular involvement in tuberculosis patients has been reported as $1.4 \%$ in a series of 10,542 patients in the U.S.A. ${ }^{5}$ Conversely, in the third world, tuberculosis is not an uncommon cause of ocular disease, in one series accounting for $30 \%$ of cases of uveitis. ${ }^{6,7}$

We report 12 cases which depict the present spectrum of disease in Great Britain, discuss the problems of diagnosis and management, and consider the possible pathogenesis.

\section{Details of Patients}

Nine patients were male, three female, aged 19-56 years (mean 35.5 years). All patients attended the Medical Eye Unit, St. Thomas' Hospital, London. Seven were from the Indian subcontinent, three from the United Kingdom, and one each from the Middle East and Africa. Nine patients presented with retinal vasculitis, two with choroidal tubercles and one with chronic anterior uveitis. The clinical details of the patients are summarised

From: The Medical Eye Unit, St Thomas' Hospital, London SE1. *Present address, Moorfields Eye Hospital, City Road, London EC1.

Correspondence to: D. J. Spalton, The Medical Eye Unit, St Thomas' Hospital, London SE1. 
Table I Ocular structures affected by tuberculosis

\begin{tabular}{lll}
\hline Structure & Sign of tuberculosis & Possible pathogenesis \\
\hline Orbit & Tuberculoma & Direct infection \\
Skin/lids & Lupus vulgaris & Direct infection \\
Lacrimal gland/sac & Tuberculoma & Direct infection \\
Conjunctivitis & Primary complex & Direct infection \\
& Tuberculoma & Direct infection \\
& Phlycten & Hypersensitivity \\
Sclera & Tuberculoma & Direct infection \\
Cornea & Phlycten & Hypersensitivity \\
Uvea & Uveitis & Direct infection/hypersensitivity \\
& Choroidal tubercle & Direct infection \\
& Iris nodules & Direct infection \\
& Endophthalmitis & Direct infection \\
Retina & Retinal vasculitis & Hypersensitivity \\
\hline
\end{tabular}

in Table II and their outcome in Table III. Four will be described in detail to illustrate the clinical spectrum of disease.

\section{Case Reports}

\section{(a) Anterior uveitis}

A 42 years old Asian female (patient 1, Table II) 18 months previously suffered an acute, undiagnosed febrile illness which completely resolved leaving her with a chronic right anterior uveitis. The left eye was normal.

On examination the right visual acuity was hand movements, the left visual acuity 6/5. Slit-lamp findings were a right anterior uveitis, corneal oedema, extensive fluffy granulomatous keratic precipitates and iris nodules (Fig. 1). The intraocular pressures were normal. No fundus details were visible due to a dense vitritis. The left eye remained entirely normal.

General examination was unremarkable, full blood count and liver function tests were normal but the ESR was $50 \mathrm{~mm} / \mathrm{hr}$. The chest X-ray was normal.

The Mantoux (1:1000) was strongly positive ( $22 \mathrm{~mm}$ induration) at $48 \mathrm{hrs}$. A presumptive diagnosis of ocular tuberculosis was made and the patient given a therapeutic trial of anti-tuberculous therapy, Rifinah 300, two daily. Within two weeks the iris nodules resolved and the uveitis had dramatically improved.

\section{(b) Choroidal tubercles}

A 37 years old Ghanaian female (patient 2, Table II) was admitted with miliary tuberculosis diagnosed by chest X-ray and confirmed by sputum culture. A course of Rifinah 300, two daily, ethambutol $1200 \mathrm{mgm}$ od and pyridoxine $10 \mathrm{mgm}$ od was commenced.

There were no ocular symptoms and she was referred for routine assessment before starting treatment. On examination the right and left visual acuities were $6 / 5$. The only abnormal features were in the fundi which showed bilateral small, white subretinal opacities with the classical appearance of choroidal tubercles (Fig. 2a). There was no evidence of retinal vasculitis. These lesions resolved with anti-tuberculous therapy for eight weeks, leaving subtle retinal pigment epithelial changes (Fig. 2b).

\section{(c) Systemic tuberculosis presenting with retinal vasculitis}

A 21 years old Arab chef (patient 6, Table II) presented to the casualty department severe visual loss in the right eye. He had a florid retinal vasculitis with a macula star and marked periphlebitis and haemorrhage. Fluorescein angiography showed marked capillary closure (Fig. $3 \mathrm{a \& b}$ ). The left eye was normal. Chest X-ray showed apical consolidation and tubercle bacilli were cultured from the sputum.

Following treatment with Ethambutol $900 \mathrm{mgm}$ od, Rifampicin $600 \mathrm{mgm}$ od, Isoniazid $300 \mathrm{mgm}$ od and Prednisolone E. C., reducing from $60 \mathrm{mgm}$ od, the ocular appearance substantially improved. Four months later he developed new vessels on the optic disc and vitreous haemorrhage. A sector panretinal photocoagulation was performed and he ultimately regained $6 / 9$ vision in this eye.

\section{(d) Retinal vasculitis followed by miliary tuberculosis}

A 35 years old Indian male (patient 12, Table II) was admitted with a five-day history of painless visual loss. There was no other relevant history. On examination the right visual acuity was $6 / 18$, left visual acuity counting fingers at 1 metre: he had a left anterior uveitis, bilateral vitritis and a bilateral retinal vasculitis. Systemic investigation was entirely normal apart from a strongly positive Man- 
Table II Systemic and Ocular involvement

\begin{tabular}{|c|c|c|c|c|c|c|}
\hline Ethnic origin & Systemic disease & Ocular disease & Diagnosis confirmed & Mantoux & $\mathbf{R x}$ & $\begin{array}{l}\text { Recent } \\
\text { infection in } \\
\text { family }\end{array}$ \\
\hline 1 India & nil & A.U. + iris nodules & nil & + & $\mathrm{T}$ & No \\
\hline 2 Ghana & Miliary & $\mathrm{Ch} \mathrm{Tb}$ & Sputum & + & $\mathrm{T}$ & No \\
\hline 3 India & Miliary & $\mathrm{Ch} \mathrm{Tb}$ & Sputum + histol & + & $\mathrm{T}$ & No \\
\hline 4 India & Pulmonary & A.U. + R.V. & Sputum + histol & + & $\mathrm{T}$ & No \\
\hline 5 U.K. & Pulmonary & A.U. + R.V. & Sputum & + & $\mathrm{T}$ & No \\
\hline 6 Arab & Pulmonary & R.V. & Chest X-ray + sputum & + & $\mathrm{T}+\mathrm{S}$ & No \\
\hline 7 India & Healed pulmonary & R.V. & Chest X-ray & + & $\mathrm{S}$ & No \\
\hline 8 U.K. & nil & R.V. & No & + & $\mathrm{T}+\mathrm{S}$ & Yes \\
\hline 9 U.K. & nil & R.V. & No & + & $\mathrm{T}+\mathrm{S}$ & No \\
\hline 10 India & nil & R.V. & No & + & $\mathrm{T}+\mathrm{S}$ & Yes \\
\hline 11 India & nil & R.V. & No & + & $\mathrm{T}+\mathrm{S}$ & No \\
\hline 12 India & & R.V. & No & + & $\mathrm{S}$ & No \\
\hline$\rightarrow$ & Miliary & $\mathrm{Ch} \mathrm{Tb}$ & $\begin{array}{l}\text { Chest X-ray + histol + } \\
\text { sputum }\end{array}$ & + & $\mathrm{T}+\mathrm{S}$ & \\
\hline
\end{tabular}

R.V.= Retinal Vasculitis, $\mathrm{T}=$ anti-tuberculous therapy, $\mathrm{Ch} \mathrm{Tb}=$ Choroidal tubercle, A.U.=Anterior uveitis, $\mathrm{S}=$ oral steroids.

toux (1:1000). Oral steroids were commenced and after two months the left and right visual acuity improved to $6 / 9$ and the vasculitis became less active, although he developed substantial retinal capillary closure.

Eight months after his initial presentation, he was admitted severely ill with miliary tuberculosis; the chest X-ray showed the typical appearances of this disease, a CT scan showed a tuberculoma in the left temporal lobe, a liver biopsy contained acid fast bacilli and caseating granulomata, and sputum, urine, and pleural fluid culture were positive for tubercle bacilli.

Ocular examination showed a resolved retinal vasculitis but multiple choroidal tubercles.

Anti-tuberculous therapy was commenced with Ethambutol $1000 \mathrm{mgm}$ od, Streptomycin $750 \mathrm{mgm}$ od, Pyridoxine $10 \mathrm{mgm}$ bd and Isoniazid $400 \mathrm{mgm}$ od. After a stormy course he made a full recovery; the right and left visual acuity improved to $6 / 5$ but is currently requiring laser photocoagulation for retinal new vessels.

\section{Discussion}

The incidence of tuberculosis although still high in the third world, has declined in the developed nations during the last century. It may not present in its classical forms to either the ophthalmologist or physician, and might not be included in a differential diagnosis of ocular disease: we feel that our recent experience with these patients reflects the present clinical spectrum of ocular pathology associated with tuberculosis.
The diagnosis of ocular involvement with tuberculosis may be considered in three ways; (a) by recovering the tubercle bacillus directly from the eye, (b) as presumed ocular disease with proven active systemic disease (c) as presumed ocular disease, without evidence of active systemic disease.

The patients reported here fall into the latter two groups: six patients had proven systemic and ocular disease, and six had ocular disease alone. In all cases the diagnosis of ocular involvement was presumptive,

Table III Outcome following treatment

\begin{tabular}{|c|c|c|}
\hline Ocular disease & $\mathbf{R x}$ & Outcome \\
\hline $1 \mathrm{Ch} \mathrm{Tb}$ & $\mathrm{T}$ & Resolved \\
\hline $2 \mathrm{Ch} \mathrm{Tb}$ & $\mathrm{T}$ & Resolved \\
\hline 3 A.U. + R.V. & $\mathrm{T}$ & Inactive \\
\hline 4 A.U. + R.V. & $\mathrm{T}$ & Inactive \\
\hline 5 R.V. & $\mathrm{T}+\mathrm{S}$ & Inactive $\rightarrow$ Laser \\
\hline $6 \mathrm{R} . \mathrm{V}$. & $\mathrm{S}$ & Inactive \\
\hline 7 R.V. & $\mathrm{T}+\mathrm{S}$ & Inactive \\
\hline 8 R.V. & $\mathrm{T}+\mathrm{S}$ & $\begin{array}{l}\text { Inactive } \rightarrow \text { Vitreous } \\
\text { Haemorrhage } \rightarrow ? \\
\text { Vitrectomy }\end{array}$ \\
\hline $\begin{array}{l}9 \text { R.V. } \\
10 \text { A.U. + iris }\end{array}$ & $\mathrm{T}+\mathrm{S}$ & Inactive $\rightarrow$ Laser \\
\hline nodules & $T+S$ & Resolved \\
\hline 11 R.V. & $\mathrm{T}+\mathrm{S}$ & Inactive \\
\hline 12 R.V. & $\mathrm{S} \rightarrow \mathrm{T}+\mathrm{S}$ & $\begin{array}{l}\text { Initially quiescent } \rightarrow \\
\text { reactivation of } \mathrm{Tb} \rightarrow \\
\text { resolved }\end{array}$ \\
\hline
\end{tabular}

$\mathrm{T}=$ anti-tuberculous therapy

$\mathrm{S}=$ systemic steroids 


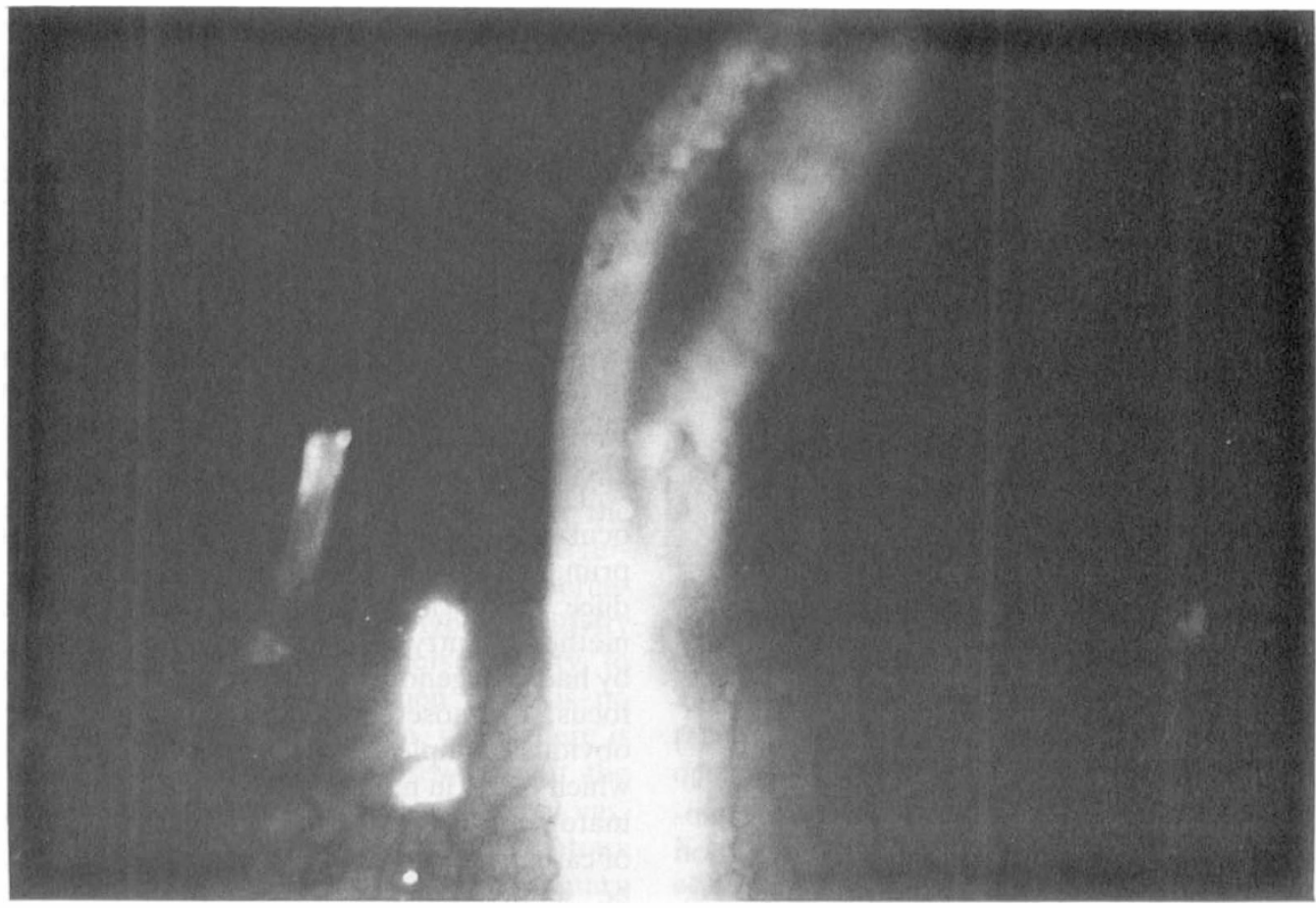

Fig. 1. Iris Nodule (case a).

direct evidence of ocular infection being impractical to obtain.

Two of our patients ( 1 and 4, Table II) demonstrate the importance of the ocular signs with the ocular disease the presenting feature of tuberculosis: the systemic disease had until this time been latent. In none of our cases did we have evidence of primary ocular tuberculosis.

The most common form of intraocular tuberculosis in this series was a retinal vas-

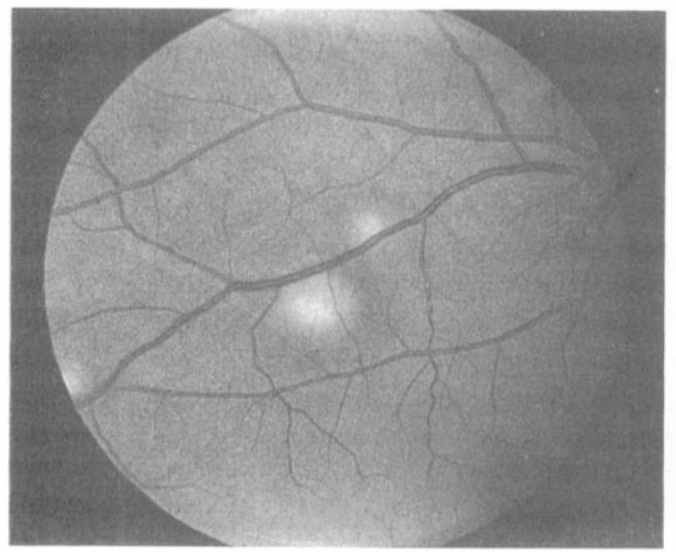

Fig. 2a. Choroidal tubercles, pre-treatment (case 1). culitis in the presence of a strongly positive Mantoux test. The characteristic features were a moderate vitreous infiltrate, severe periphlebitis, and a marked tendency to peripheral retinal capillary closure leading to new vessel formation. The latter was a particularly noticeable feature occurring in four $(25 \%)$ of our patients. In comparison with sarcoidosis, ${ }^{9}$ peripheral closure appears to be more common in ocular tuberculosis with peripheral retinal new vessels predominating. Sector

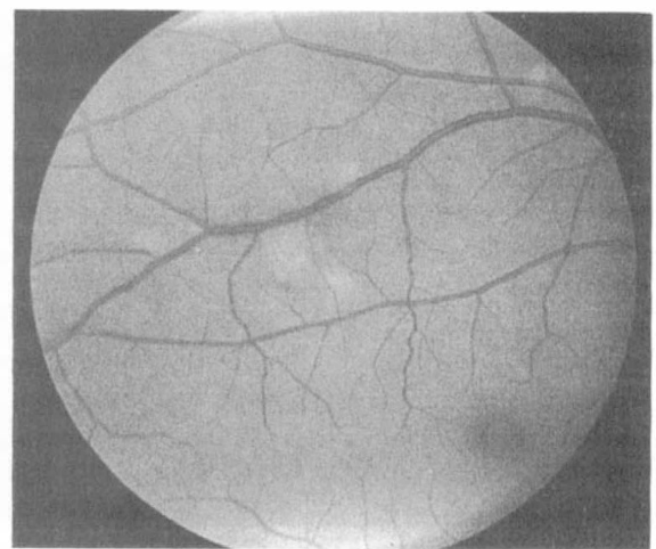

Fig. 2b. Choroidal tubercle, post treatment (case 1). 


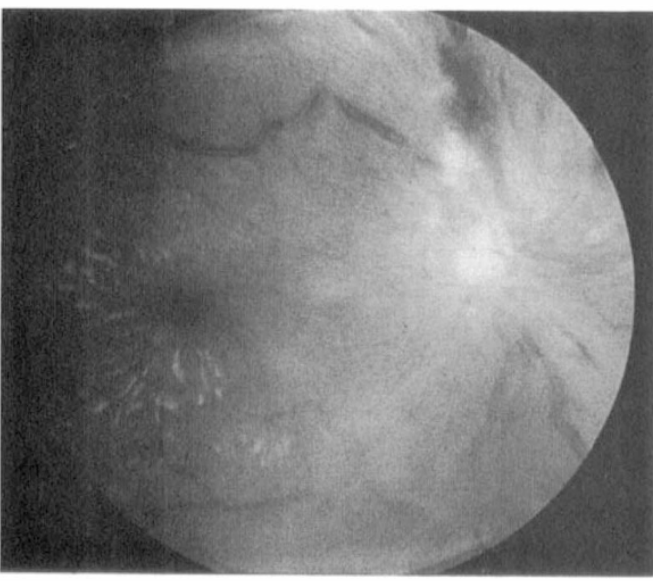

Fig. 3a. Retinal vasculitis and macular star (case 6).

pan-retinal photocoagulation has been successfully used by us (patients 4 and 7, Table II) and others in treating this complication. ${ }^{10}$

The pathogenesis of intraocular tuberculosis has received comparatively little attention since the first half of this century. ${ }^{11-13}$ The original experiments involved the injection of either live or heat killed bacilli onto or into the rabbit eye. Finoff ${ }^{11,12}$ concluded that inoculation of the bacilli was needed to produce ocular tuberculosis and that, interestingly, intracarotid injection of either living or dead bacilli could produce uveitis, choroidal tubercle and rarely retinal vasculitis in rabbits. Verhoeff demonstrated the presence of a tubercle bacillus in the central retinal vein of a patient with 'periphlebitis retinae'. ${ }^{14}$ These experiments leave doubt as to whether the ocular signs are due to active infection with bacteria or a hypersensitivity response. Clinically intraocular tuberculosis can be due to 'direct infection' or 'indirect' (hypersensitivity) mechanisms (Table I).

'Direct' refers to the live bacillus causing ocular disease. Finoff ${ }^{11}$ concluded that primary extra-ocular infection could not produce intraocular disease. Therefore the method of entry into the globe is thought to be by haematogenous spread from a tuberculous focus, diagnosed or occult, elsewhere. An obvious example of this is choroidal tubercles which occur in miliary tuberculosis from haematogenous spread and histologically consist of caseating granulomata in which bacilli may be seen. Similarly the reported cases of endophthalmitis ${ }^{15}$ are caused by direct infection. A rapid clinical response to anti-tuberculous therapy would seem likely to indicate that the ocular disease is due to direct infec-

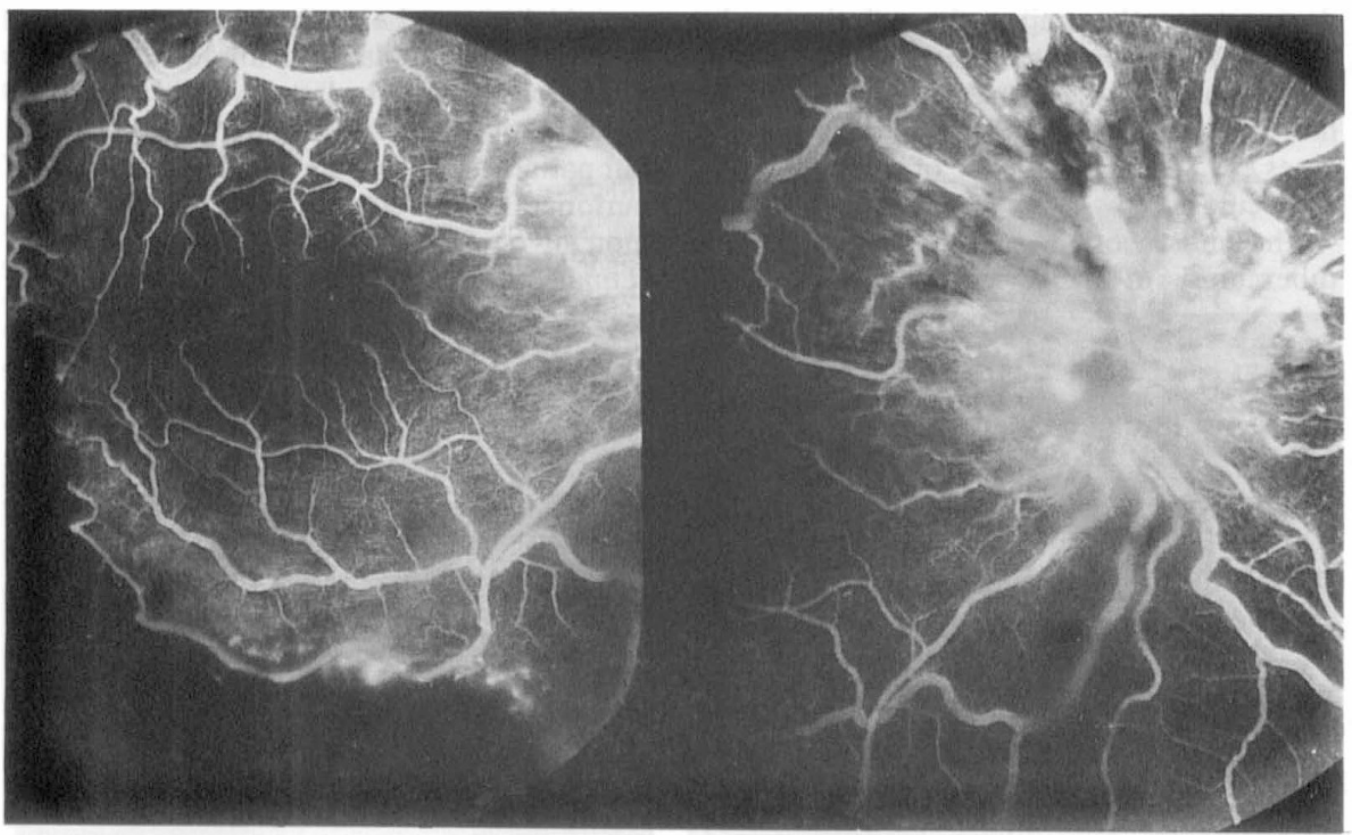

Fig. 3b. Peripheral retinal capillary closure (case 6). 
tion and this was the case in three of our patients with anterior uveitis and choroidal tubercles (1, 2 and 3, Table II). It also forms the basis of Schlaegel's isoniazid therapeutic test in uveitis. ${ }^{16}$

The pathogenesis of indirect involvement of the eye, where hypersensitivity and immunological mechanisms are important, is less clear: the intraocular clinical entities in this group are uveitis and retinal vasculitis, but may also be reflected in other series as scleritis and conjunctival phylyctens. Retinal vasculitis has frequently been circumstantially associated with tuberculosis ${ }^{17,18}$ however the question is unresolved as to whether the retinal vasculitic lesions contain active tuberculous bacilli or reflect a hypersensitivity response. Theoretically hypersensitivity to tuberculosis could occur when there is no defined active systemic lesion, when there is an active systemic lesion elsewhere or the lesion is thought to be inactive. Retinal vasculitis has been reported after a Mantoux test $^{19}$ and in association with alimentary tuberculosis ${ }^{20,21}$ and it may be that Asian patients are more susceptible to gastro-intestinal tuberculosis, although we did not look for this in our series.

Of our patients in this group with retinal vasculitis, three had active systemic disease and seven no overt active disease, although all had strongly positive Mantoux tests, a high erythrocyte sedimentation rate or positive family history. One patient (number 12; Table II) is of particular interest. He initially had retinal vasculitis with no apparent active systemic disease, but after treatment with oral steroids, he developed florid miliary tuberculosis. This suggests that his retinal vasculitis, which might be due to a hypersensitivity response, had responded to systemic steroids to be replaced by choroidal tubercles in the presence of active/reactivated infection.

The definitive diagnosis of tuberculosis and intraocular tuberculosis is often difficult. It is important to have an index of suspicion of the diagnosis in patients with atypical anterior uveitis, choroidal lesions or retinal vasculitis, and this will be influenced by the socio-economic circumstances, family history, ethnic origin and previous medical history of the patient. Ocular examination will also provide clues such as an intense granulomatous anterior uveitis poorly responsive to steroid treatment, iris nodules, angle granulomata or choroidal tubercles.

Tuberculous retinal vasculitis should be suspected in the presence of a florid retinal vasculitis with marked capillary closure with a relatively mild degree of vitreous cellular infiltrate, particularly in patients of Asiatic origin: genetic predisposition may account for the propensity to develop retinal vasculitis in these patients in contrast to Caucasians.

It is obviously essential to exclude other causes of ocular inflammation, such as sarcoidosis, at the same time as looking closely for evidence of systemic tuberculosis (Fig. 4). In nearly all reported cases, the diagnosis of intraocular tuberculosis is only presumptive. The presence of active or inactive extraocular tuberculous infection does not prove intraocular tuberculosis.

In the investigation of these patients a chest X-ray should routinely be performed although it may be normal.

The Mantoux test provides a simple and easily performed investigation which is relatively specific. ${ }^{22-25}$ However the antigens in the PPD cross react with atypical mycobacteria

\section{Systemic Infection}

\begin{tabular}{|c|c|}
\hline \multicolumn{2}{|l|}{ Chest X - Ray } \\
\hline Mantoux & \\
\hline $\begin{array}{l}\text { Sputum } \\
\text { Urine } \\
\text { Faeces }\end{array}$ & Culture \\
\hline $\begin{array}{l}\text { Lymph Nodes } \\
\text { Liver }\end{array}$ & $\begin{array}{l}\text { Biopsy, } \\
\text { Culture }\end{array}$ \\
\hline
\end{tabular}

\section{Ocular Infection}

\begin{tabular}{l|l} 
Aqueous & Culture \\
Vitreous & \\
Triple therapy therapeutic test \\
Histology
\end{tabular}

Fluorescein Angiography

Fig. 4. Investigation of suspected Ocular Tuberculosis. 
and incorrect technique can lead to either false positives or negative results. The picture is further complicated by the fact that in Great Britain, unlike the United States, most patients have received BCG and therefore will show a hypersensitivity response to PPD. ${ }^{22}$

The definitive systemic diagnosis is made by culture of M. tuberculosis from body fluids or biopsy material. It is important to have positive cultures and sensitivities to allow the instigation of appropriate chemotherapy. Bronchoscopy, gastric lavage, liver biopsy, lymph node biopsy or faecal culture must be performed as required by the clinical history and the results of haematological investigation.

The management of these patients can be considered in two parts. Firstly management of the systemic disease, with standard antituberculous therapy, and secondly, management of the ocular disease. If retinal vasculitis is treated alone with systemic steroids it is possible to induce a reactivation of the systemic illness as occurred with our patient, (2, Table II) and we strongly suggest that if tuberculous retinitis is suspected appropriate chemotherapy should be given even in the absence of active systemic disease to avoid this complication. New vessel formation associated with retinal vasculitis and retinal capillary closure responds to laser photocoagulation.

The incidence of tuberculosis is declining and it is easily missed in its extrapulmonary form. It is a readily treatable disease and the consequences of delay in either ocular or systemic diagnosis can be very serious for the patient. It should be considered by the ophthalmologist when unexplained chronic anterior uveitis, iris nodules and retinal vasculitis occur. Similarly, the physician should not forget that the ophthalmologist can confirm the clinical diagnosis of miliary tuberculosis and sometimes support the diagnosis of the other forms of systemic infection.

The authors wish to thank Mr M. D. Sanders for allowing us to report his patients and Dr N. Bateman for his helpful comments.

\section{References}

${ }^{1}$ Dinning WJ and Marston S: Cutaneous and Ocular Tuberculosis. J Roy Soc Med 1985, 78: 576-81.

${ }^{2}$ James $G$ and Mishra B: The Changing Pattern of Tuberculosis. Postgrad Med J 1984, 60: 14-19.

${ }^{3}$ Woods AC: Endogenous inflammations of the uveal tract. Williams and Wilkins, 1961.

${ }^{4}$ Schlaegel RF and O'Connor GR: Metastatic nonsuppurative uveitis. Int Ophthalmol Clin 1977, 17: 87-108.

${ }^{5}$ Donahue HC: Ophthalmologic Experience in a Tuberculosis Sanatorium. Am J Ophthalmol 1967, 64: 742-8.

${ }^{6}$ Sen KD: Endogenous Uveitis in Indian Children. $J$ Paed Ophthalmol, 14: 25-31.

${ }^{7}$ Consul BN, Sharma DP, Chalabra MS, Sahai R: Uveitis: Aetiological pattern in India. Eye, Ear, Nose, Throat Monthly 1972, 51: 122-6.

${ }^{8} \mathrm{Ni}$ C, Papale JJ, Robinson N, Wu B: Uveal Tuberculosis. Int Ophthalmol Clin 1982, 22: 103-24.

${ }^{9}$ Spalton DJ and Sanders MD: Fundus changes in histologically proven sarcoid. Br J Ophthalmol 1981, 65: 348-58.

${ }^{10}$ Gur S, Siverstone B, Zylberman R, Berson D: Chorioretinitis and Extrapulmonary Tuberculosis. Ann Ophthalmol 1987, 19: 112-15.

${ }^{11}$ Finoff WC: Ocular tuberculosis, experimental and clinical. Arch Ophthalmol 1923, 53: 130-6.

${ }^{12}$ Finoff WC: Changes found in eyes of rabbits following injection of tubercle into the common carotid artery. Am J Ophthalmol 1924, 7: 81-9.

${ }^{13}$ Ohmart WA: Experimental Tuberculosis of the Eye. Am J Ophthalmol 1933, 16: 773-8.

${ }^{14}$ Verhoeff FH and Simpson GV: Tubercle within the central retinal vein. Arch Ophthalmol 1940.

${ }^{15}$ Darell RW: Acute Tuberculous Panophthalmitis. Arch Ophthalmol 1967, 78: 58-9

${ }^{16}$ Abrams J and Schlaegel TF: The Role of the Isoniazid Therapeutic Test in Tuberculous Uveitis. Am J Ophthalmol 1982, 94: 511-15.

${ }^{17}$ Fountain JA and Werner R: Tuberculous Retinal Vasculitis. Retina 1984, 4: 48-50.

${ }^{18}$ Elliot AJ: Thirty year Observation of Patients with Eales Disease. Am J Ophthalmol 1975, 80: 404-8.

${ }^{19}$ Muncaster S: Bilateral uveitis and periarteritis as a focal reaction to the tuberculin test. Arch Ophthalmol 1939, 21: 509-10

${ }^{20}$ Taylor OG: Longstanding Presumed Tuberculous Ocular Allergy of Alimentary Origin. Ann Ophthalmol 1974, 6: 229-33.

${ }^{21}$ Seward DN: Tuberculoma of the Ciliary Body. Med J Aust. 1973, 1: 298-8.

${ }^{22}$ Glassroth J, Robins AG, Snider DE: Tuberculosis in the 1980s. $N$ Eng J Med 1980, 302: 1441-50.

${ }^{23}$ Gantz NM: Tuberculin skin testing: when and how to use it. Medical Practice 1986, Sept: 25-33.

${ }^{24}$ Abrams J and Schlaegel TF: The Tuberculin Skin Test in the Diagnosis of Tuberculous Uveitis. $A m$ J Ophthalmol 1982, 96: 295-8.

${ }^{25}$ Barclay WR: Does a positive tuberculin test indicate presence of live tubercle bacilli. J Am Med Ass 1975, 232: 755-8 